\title{
DIRECTIONAL WELL TRAJECTORY DESIGN: THE THEORITICAL DEVELOPMENT OF AZIMUTH BENDS AND TURNS IN COMPLEX WELL TRAJECTORY DESIGNS
}

\author{
P. 0. Okpozo ${ }^{1}$, A. Peters ${ }^{2}$ and W. C. Okologume ${ }^{3}$ \\ 1,2, 3 Dept. of Petroleum and Nat. Gas Engr., Federal Univ. of Pet. Resources, Effurun, Delta State, Nigeria. \\ E-mail addresses: ${ }^{1}$ okpozo.paul@fupre.edu.ng, ${ }^{2}$ petersakpoturi1212@gmail.com, \\ 3 okologume.wilfred@fupre.edu.ng
}

\begin{abstract}
Most complex well trajectories usually have bends and turns. The design requires some considerations to be made from the structure of subsurface formations to be drilled, to the available technology and equipment, as well as economics. When a complex well trajectory is being worked on, the effect of bends/turns in the azimuth is highly critical and care is taken not to exceed a set maximum tolerable dogleg severity. In this work, several well trajectories with varying bends and turns were modelled mathematically, for an easy calculation and pre-survey record presentation of a desired complex directional well trajectory, also from which the overall angle change (dogleg) is observed not to exceed a set maximum tolerable dogleg. A scenario was considered with few assumptions using a specially developed application, WellTIT v. 1.0, which assisted in calculating and presenting survey records to express the need for carefully developed bends in a Complex well trajectory design.
\end{abstract}

Keywords: azithuth, bends, bottom hole assembly, complex directional well, trajectory well

\section{INTRODUCTION}

Directional drilling is a process of directing a well trajectory to some pre-planned target intentionally [1, 2].

This is done under the consideration of effective economical application of both time and finance, as well as a means of managing subsurface challenges. Speaking of subsurface challenges, this could be;

1) Existing wells (part of field plan)

2) Relief wells (to kill a subsurface blowout well)

3) Sidetracking (due to a breakage and loss of drill string section in the hole)

4) Pocket reservoirs that could be commingled and produced using one well.

In modern times the need to drill directionally has increased because of its economics and improved efficiency in its technology, such wells are planned and drew in two-dimensional form, as well as 3dimensional form. Lubinski, was actually one of the pioneers to introduce mathematics into directional well trajectory plan [3]; which has led to a wellstructured and detailed trajectory plans. This in turn has led to the development of well planning softwares that are being used today.

One main aim in directional well trajectory plan is the development of a mathematical model that will best represent a given well path. There are three (3) basic directional well trajectory designs; Build-and-Hold trajectory, Build-Hold-and-Drop trajectory and Continuous build trajectory, whose mathematical models are presented across some drilling technology texts [1-5]. These models were modelled using straight lines in the horizontal departure/Azimuth direction. Meanwhile most complex models use the shape of a sphere and/or cylinder to describe curve between two points [3].

Such bent curves are identified as Turns in drilling technology terms, which give rise to a complex mathematical model, slightly modified and different from the basic models; the basis is shown in the next section (Section 2.1). These turns are associated with the azimuth direction coordinate system (North, North-East, East, South-East, South, South-West, West and North-West) where the North and South coordinates are reference measure start points. For 
example; $23^{\circ} \mathrm{NE}$ means 23 degrees East from North, $35^{\circ} \mathrm{SW}$ means 35 degrees West from South.

Also, from observation on most complex well trajectory designs, we have seen that they could be dissected into sections of either two or more of the basic well model trajectories; these are combined in a highly sophisticated form, and if each could be carefully modelled and combined could produce a well-designed mathematical trajectory model. An example is shown in figure (1) below, which can be seen to consist of two basic trajectories:

1. The Build and Hold with a TURN (NE direction), from 0 to 2250 meters TVD.

2. A Straight course tangent to Build and Hold (SE direction), from 2250 to 2800 meters TVD.

As bends and turns are planned for and designed in a well, critical consideration is given to the maximum tolerable dogleg severity. Firstly, Dogleg angle is the change in overall angle between two survey station points. Overall angle change has to do with the combination of change in inclination angle $\left(\Theta_{1-2}\right)$ and change in Azimuth $\left(\emptyset_{1-2}\right)$ between points 1 and 2 . Dogleg severity on the other hand is the Dogleg angle per unit course length[6]. Dogleg severity is directly linked to the bending force of the pipe. Drill pipes, Casings, wellbore sweeping efficiency, wellbore stability, well logging and safety factors related to stuck pipe do generate a set value for a maximum tolerable dogleg severity. Even while in drilling operation, careful designs are made for the directional drilling tools and techniques which are used to change or build an inclination angle and/or Azimuth of the well path. Often times, the bit is oriented to face a proposed angular direction (in most survey records, it is termed as tool face angle) and then with an applied force on the bit [7]. The Bottom Hole Assembly (BHA) is what provides this necessary effect. Steering tools gives the directional drilling operator the information on where to situate the tool face angle and achieve better control. One of such steering tools is the Measurement While Drilling (MWD) electronic tool. Though this surveying tool is operated in response to the geomagnetic effect (Magnetic REFERENCE), and the effect caused by the drill collar (Magnetic INTERFERENCE).

The influence caused by the MAGNETIC REFERENCE, a mathematical model (Global Geomagnetic Model) of the earth's magnetic field in its undisturbed state, developed by the British Geological Survey (BGS) [8], programmatically help the MWD survey tools measure the direction of a well path relative to the direction of the geomagnetic field. The difference between the geomagnetic north and the geographic (true) north is the DELINEATION ANGLE. This delineation angle changes with time and it depends on the position and surface features of the earth. This is the reason why the data from BGS is updated yearly. Most drilling companies and operators, even Halliburton and Schlumberger subscribe to this data provision by BGS to update their software programs for improved accuracy.

As for the effect caused by MAGNETIC INTERFERENCE, non-magnetic drill collars interfere on surveying instruments (MWD), that is why a required size of drill collar is selected in relation to the location of the wellbore in the earth, it's inclination and direction (angle readings from North or South), as well as zone in the earth's surface. An empirical relationship can be seen in [1]-page (393).

The weight or size of drill collar should be carefully selected because excessive weight on the bit might lead to increase in reverse torque, and thereby causing the tool face rotating/drifting back towards the right as the bit drills off.

Some common deflecting tools for directional drilling are;

- Positive Displacement Motors (PDM) with Bent Sub - This method operates by only the bit rotating while the drill string remains static. It is often referred to as the sliding technique. The Bent sub handles the deflection or bit orientation. Though, this often causes stuck pipe problems, as well as difficulty in maintaining well path.

- Rotary Steerable System (RSS) - This method operates by both the bit and drill string rotating except the BHA unit which handles the deflection or orientation of the bit. This tool is mostly preferred in terms of its wellbore and accuracy.

- Whipstock - This technique uses a slant deflecting equipment installed in the hole to deflect the entire drill string when encountered. This is often used to sidetrack the well path from a plugged hole or wrong hole direction. etc.

Since well plan is an essential part of drilling technology, software models are created and comprises various calculation methods which are used to find exact and true coordinates for a well path. When constructing a directional well trajectory path, the functions in the software model gives room for the user to input various values and selected parameters to get the desired well trajectory path and coordinates. One of such leading planning tool is the 
Halliburton's COMPASS; a powerful planning tool, although with limited public information on its functions and built, as well as mathematical calculation models utilized in its built. Also, because of its cost and affordability in Nigerian universities dealing on the topic of directional drilling, there have been several motives to develop well trajectory design calculation models to help educate students on the topic. The use of Microsoft Excel Sheet [9] is most used by lecturers to solve robust mathematical problems, but this is not quite enough. Stromhaug [3] tried designing modules for handling well trajectory design problems using COMPASS to calibrate His designed models. We took this initiative to develop WellTIT (Well Trajectory Illustration Tool) version 1.0, which consist of several derived mathematical trajectory models that could be used to model both basic and complex well trajectories. WellTIT v. 1.0 is our tool to test our derived mathematical theory for its accuracy by using Radius of Curvature survey calculation method.

The aims of this work are;

- Firstly, to create a mathematical theory for curved well departures.

- Secondly, the idea that governs combination of basic trajectories to form a complex well system.

- Thirdly, the mathematical theory that guides careful combination of different azimuth bends and tangents in a complex well system.

- Lastly, the introduction and application of computer model (WellTIT) to prove one of the theory's validity.

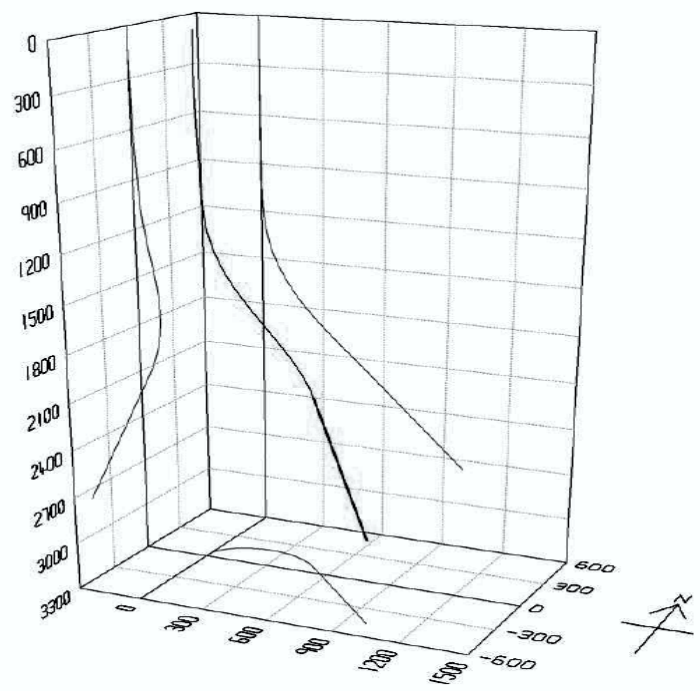

Figure 1: COMPASS well path complex trajectory plot (courtesy Andreas Stromhaug, 2014)
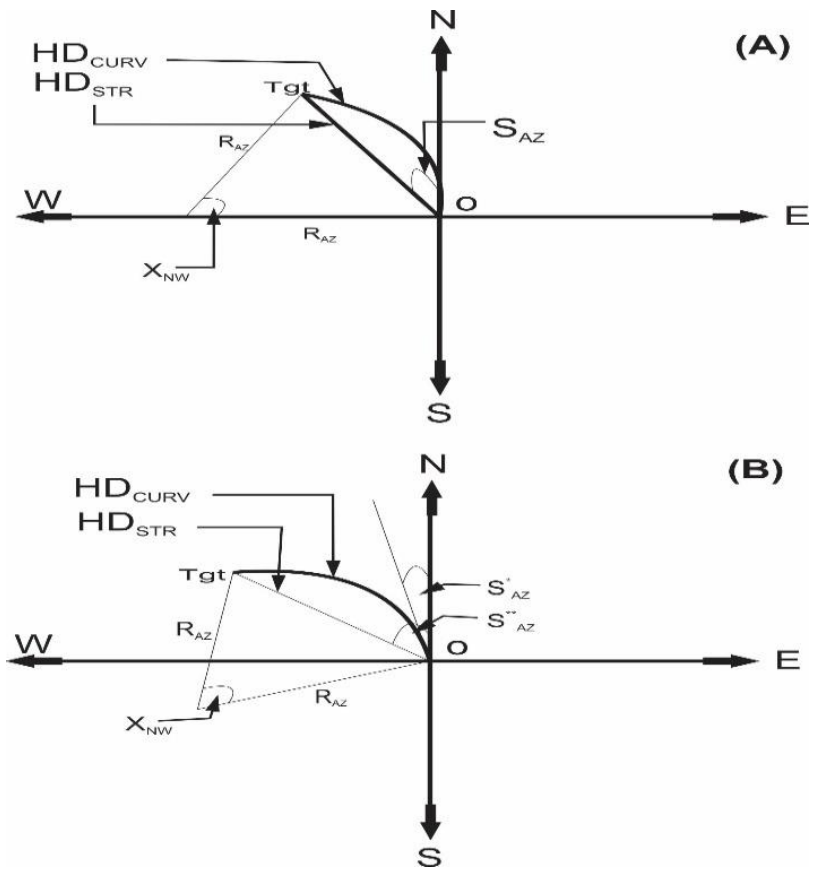

Figure 2: Diagrammatic representation of variables in an azimuth curve illustration

\section{THEORY AND DESCRIPTIONS}

\subsection{Azimuth Curve Bend (Turn) Basic Illustration Model}

Observing the above figure (2), assuming a certain trajectory is deviated from point 0 and drilled to a target $\mathrm{T}_{\text {gt. }}$ The direct/straight line horizontal departure, $\mathrm{HD}_{\mathrm{STR}}$, is known and an azimuth deviation, $S_{A Z}$. In figure (2-A), the deviation azimuth tends $S_{A Z^{\circ}}$

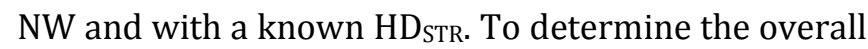
azimuth angle change $\mathrm{X}_{\mathrm{NW}}$, in triangle $\Delta \mathrm{OMT}_{\mathrm{gt}}$, and the azimuthal radius of curvature, $\mathrm{R}_{\mathrm{AZ}}$, and thence the curved horizontal departure, $\mathrm{HD}_{\mathrm{CURv}}$, goes this way;

$$
X_{N W}=2 \times S_{A Z}
$$

From general mathematical formula used for solving angles and lines in triangles, we use the cosine rule,

$$
\begin{aligned}
& H D_{S T R}^{2}=R_{A Z}^{2}+R_{A Z}^{2}-2 \times R_{A Z} \times R_{A Z} \times \operatorname{Cos}\left(X_{N W}\right) \\
& R_{A Z}=\left\{\frac{H D_{S T R}^{2}}{2\left[1-\operatorname{Cos}\left(X_{N W}\right)\right]}\right\}^{1 / 2} \\
& H D_{C U R V}=\frac{X_{N W} \times \pi \times R_{A Z}}{180} \text { OR } \frac{S_{A Z} \times \pi \times R_{A Z}}{90}
\end{aligned}
$$

For figure (2-B), the azimuth deviation kicked off from a certain deviated angle, $S_{\mathrm{AZ}}{ }^{*}$, which ended at $\mathrm{S}_{\mathrm{AZ}}{ }^{* *}$ and target ' $\mathrm{T}_{\mathrm{gt}}$ '. Due to the tilt created by $\mathrm{S}_{\mathrm{AZ}}{ }^{*}$ that affected the triangle, $\triangle \mathrm{OMT}_{\mathrm{gt}}$, the entire solution becomes;

$$
\begin{aligned}
& X_{N W}=2 \times\left(S_{A Z}^{* *}-S_{A Z}^{*}\right) \\
& H D_{C U R V}=\frac{X_{N W} \times \pi \times R_{A Z}}{180} \\
& =\frac{\left(S_{A Z}^{* *}-S_{A Z}^{*}\right) \times \pi \times R_{A Z}}{90}
\end{aligned}
$$



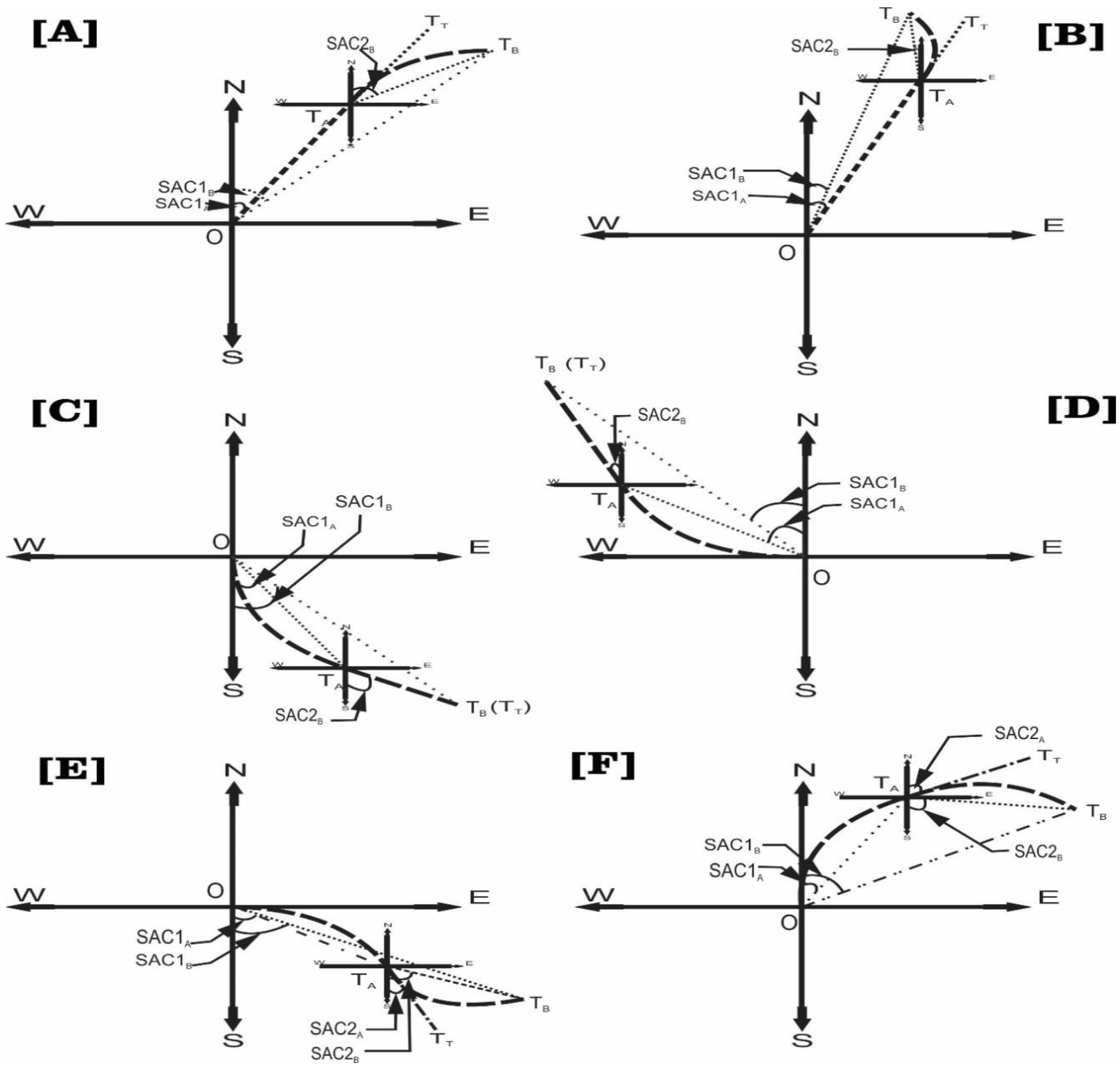

Figure 3: Diagrammatic plots of complex wells azimuth bends and their mathematical illustration

\subsection{Mathematical Model of Complex Well Trajectories With Multiple Azimuth Bends/Turns}

Figure $3\{(\mathrm{~A})$ to $(\mathrm{F})\}$ shows some complex trajectory designs from the horizontal and azimuth direction view. The azimuth survey is read from the North or South, i.e. NE, SE, SW and NW [4]. The meanings of the representations in Figure 3 is shown in the nomenclature below.

\subsubsection{Straight Azimuth departure course to Curved Azimuth departure course (Fig.3[A]\&[B])}

Observing the above mentioned figures, assuming a complex trajectory starts from a straight course and ends with a curved course. With all necessary information available, and application of the theory in section (2.1) above, we are left to determine the straight line horizontal departure of the second course, $\overline{T_{A} T_{B}}$.

Let, $m=T_{A} \hat{O} T_{B}, v=O \widehat{T_{A}} T_{B}, u=T_{T} \widehat{T_{A}} T_{B}$.

The determination of ' $\mathrm{v}$ ' and ' $\mathrm{m}$ ' is shown in Table 1. Also, utilizing the triangle, $\Delta O T_{A} T_{B}$, from which we can determine line, $\overline{T_{A} T_{B}}$. Having the departure distance of the first trajectory ' $\mathrm{A}$ ', $\overline{O T_{A}}$, and overall target ' $\mathrm{T}_{\mathrm{B}}$ ' distance from origin ' $O$ ', $\overline{O T_{B}}$, we use the Sine rule;

$$
\frac{\overline{O T_{A}}}{\operatorname{Sin}(v)}=\frac{\overline{T_{A} T_{B}}}{\operatorname{Sin}(m)}
$$

For the second trajectory course, we use ' $\mathrm{u}$ ' and $\overline{T_{A} T_{B}}$ into the theorem in section 2.

2.2.2 Curved Azimuth departure course to Straight Azimuth departure course (Fig.3[C]\&[D])

Vol. 35 No. 4, October, 2016 
Observing the titled figures, assuming a complex trajectory starts from a curved course and ends with a straight course along the tangent of the curved course. Taking the representative variables from (a) above and the determination of ' $m$ ' and ' $v$ ' from Table 2, the straight course, $\overline{T_{A} T_{B}}$, can be determined using equation (2).

\subsubsection{Curved Azimuth departure course to Curved Azimuth departure course [OPPOSITE BEND DIRECTION] (Fig.3[E])}

Observing the titled figure, assuming a complex trajectory starts from a curved course bent toward a certain direction and the other curved course starts from the tangent of the first curve while bent toward the opposite direction of the first. Taking the representative variables from (a) above and the determination of ' $\mathrm{m}$ ' and ' $\mathrm{v}$ ' from Table 4 , the straight course, $\overline{T_{A} T_{B}}$, can be determined using equation (2).
The entire course length for both curves can be solved using the theorem explained in section (2.1)above, and combining both together.

\subsubsection{Curved Azimuth departure course to Curved Azimuth departure course [SAME BEND DIRECTION] (Fig.3[F])}

Observing the titled figure, assuming a complex trajectory starts from a curved course bent toward a certain direction and the other curved course starts from the tangent of the first curve while bent toward the same direction of the first. Taking the representative variables from (1) above and the determination of ' $m$ ' and ' $v$ ' from Table 3, the straight course, $\overline{T_{A} T_{B}}$, can be determined using equation (2). The entire course length for both curves can be solved using the theorem explained in section (2.1) above, and combining both together.

Table 1: Determination of ' $v$ ' and ' $m$ ' Straight Azimuth departure course to Curved Azimuth departure course

\begin{tabular}{|c|c|c|c|}
\hline \multicolumn{4}{|c|}{ Azimuth Angles Selection } \\
\hline $\begin{array}{c}\text { SAC } 1_{A} \text { (First Trajectory } \\
\text { Azimuth Direction) - } \\
\text { FROM }\end{array}$ & $\begin{array}{c}\text { SAC } 2_{\mathrm{B}}(\text { Second } \\
\text { Trajectory Azimuth } \\
\text { Direction })- \text { TO }\end{array}$ & V & M \\
\hline NW & $\mathrm{NE}$ & $180-\left(\mathrm{SAC} 1_{\mathrm{A}}+\mathrm{SAC} 2_{\mathrm{B}}\right)$ & Abs $\left(S A C 1_{A}-S A C 1_{B}\right)$ \\
\hline NE & NW & $180-\left(\mathrm{SAC} 1_{\mathrm{A}}+\mathrm{SAC} 2_{\mathrm{B}}\right)$ & Abs $\left(S A C 1_{A}-S A C 1_{B}\right)$ \\
\hline SW & SE & $180-\left(\mathrm{SAC} 1_{\mathrm{A}}+\mathrm{SAC} 2_{\mathrm{B}}\right)$ & Abs $\left(S A C 1_{A}-S A C 1_{B}\right)$ \\
\hline SE & SW & $180-\left(\mathrm{SAC} 1_{\mathrm{A}}+\mathrm{SAC} 2_{\mathrm{B}}\right)$ & Abs $\left(S A C 1_{A}-S A C 1_{B}\right)$ \\
\hline NW & $\begin{array}{c}\text { NW (AntiClockwise) } \\
\text { NW (Clockwise) }\end{array}$ & $\begin{array}{l}S A C 1_{A}+\left(180-S A C 2_{B}\right) \\
S A C 2_{B}+\left(180-S A C 1_{B}\right)\end{array}$ & $\operatorname{Abs}\left(S A C 1_{A}-S A C 1_{B}\right)$ \\
\hline NE & $\begin{array}{l}\text { NE (AntiClockwise) } \\
\text { NE (Clockwise) }\end{array}$ & $\begin{array}{l}S A C 2_{B}+\left(180-S A C 1_{B}\right) \\
S A C 1_{A}+\left(180-S A C 2_{B}\right)\end{array}$ & Abs $\left(S A C 1_{A}-S A C 1_{B}\right)$ \\
\hline SE & $\begin{array}{l}\text { SE (AntiClockwise) } \\
\text { SE (Clockwise) }\end{array}$ & $\begin{array}{l}S A C 1_{A}+\left(180-S A C 2_{B}\right) \\
S A C 2_{B}+\left(180-S A C 1_{B}\right)\end{array}$ & $\operatorname{Abs}\left(S A C 1_{A}-S A C 1_{B}\right)$ \\
\hline SW & $\begin{array}{c}\text { SW (AntiClockwise) } \\
\text { SW (Clockwise) }\end{array}$ & $\begin{array}{l}S A C 2_{B}+\left(180-S A C 1_{B}\right) \\
S A C 1_{A}+\left(180-S A C 2_{B}\right)\end{array}$ & $\operatorname{Abs}\left(S A C 1_{A}-S A C 1_{B}\right)$ \\
\hline NW & SW & $\mathrm{SAC} 1_{\mathrm{A}}+\mathrm{SAC} 2_{\mathrm{B}}$ & $\operatorname{Abs}\left(\mathrm{SAC} 1_{\mathrm{A}}-\mathrm{SAC} 1_{\mathrm{B}}\right)$ \\
\hline SW & NW & $S A C 1_{A}+S A C 2_{B}$ & Abs $\left(S A C 1_{A}-S A C 1_{B}\right)$ \\
\hline SE & NE & $S A C 1_{A}+S A C 2_{B}$ & Abs $\left(S A C 1_{A}-S A C 1_{B}\right)$ \\
\hline NE & SE & $S A C 1_{A}+S A C 2_{B}$ & $\operatorname{Abs}\left(S A C 1_{A}-S A C 1_{B}\right)$ \\
\hline
\end{tabular}

Table 2: Determination of ' $v$ ' and ' $m$ ' Curved Azimuth departure course to Straight Tangent Azimuth departure course

\begin{tabular}{|c|c|c|c|c|}
\hline \multicolumn{5}{|c|}{ Azimuth Angles Selection } \\
\hline $\begin{array}{c}\text { SAC1 }_{A}(\text { First } \\
\text { Trajectory Azimuth } \\
\text { Direction) - FROM }\end{array}$ & $\begin{array}{c}\mathrm{SAC} 2{ }_{\mathrm{B}}(\text { Second } \\
\text { Trajectory } \\
\text { Azimuth Direction }) \\
\text { - T0 }\end{array}$ & $\begin{array}{l}\mathrm{SAC}_{\mathrm{B}}(\text { Azimuth } \\
\text { deviation of final } \\
\text { target from the } \\
\text { origin) }\end{array}$ & V & $\mathrm{m}$ \\
\hline SE (Clockwise) & \multirow{2}{*}{$\mathrm{SE}$} & \multirow{2}{*}{ SE } & $\begin{array}{c}\mathrm{SAC} 2_{\mathrm{B}}+(180- \\
\left.\mathrm{SAC} 1_{\mathrm{B}}\right)\end{array}$ & \multirow{2}{*}{$\operatorname{Abs}\left(S A C 1_{A}-S A C 1_{B}\right)$} \\
\hline SE (Anticlockwise) & & & $\begin{array}{c}\mathrm{SAC} 1_{\mathrm{A}}+(180- \\
\left.\mathrm{SAC} 2_{\mathrm{B}}\right)\end{array}$ & \\
\hline NW(Clockwise) & \multirow{2}{*}{ NW } & \multirow{2}{*}{ NW } & $\begin{array}{c}\mathrm{SAC} 2_{\mathrm{B}}+(180- \\
\left.\mathrm{SAC} 1_{\mathrm{B}}\right)\end{array}$ & \multirow{2}{*}{$\operatorname{Abs}\left(S A C 1_{A}-S A C 1_{B}\right)$} \\
\hline NW(Anticlockwise) & & & $\begin{array}{c}\mathrm{SAC} 1_{\mathrm{A}}+(180- \\
\left.\mathrm{SAC} 2_{\mathrm{B}}\right)\end{array}$ & \\
\hline
\end{tabular}




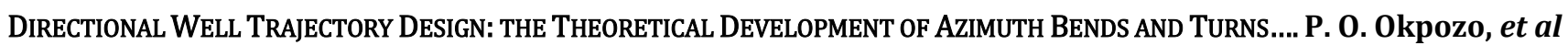

\begin{tabular}{|c|c|c|c|c|}
\hline \multicolumn{5}{|c|}{ Azimuth Angles Selection } \\
\hline $\begin{array}{c}\text { SAC1 } 1_{A}(\text { First } \\
\text { Trajectory Azimuth } \\
\text { Direction) - FROM }\end{array}$ & $\begin{array}{c}\mathrm{SAC} 2_{\mathrm{B}}(\text { Second } \\
\text { Trajectory } \\
\text { Azimuth Direction }) \\
\text { - TO }\end{array}$ & $\begin{array}{l}\mathrm{SAC}_{\mathrm{B}}(\text { Azimuth } \\
\text { deviation of final } \\
\text { target from the } \\
\text { origin) }\end{array}$ & $\mathrm{V}$ & $\mathrm{m}$ \\
\hline SW(Clockwise) & \multirow{2}{*}{ SW } & \multirow{2}{*}{ SW } & $\begin{array}{c}\mathrm{SAC} 1_{\mathrm{A}}+(180- \\
\left.\mathrm{SAC} 2_{\mathrm{B}}\right)\end{array}$ & \multirow{2}{*}{$\operatorname{Abs}\left(S A C 1_{A}-S A C 1_{B}\right)$} \\
\hline $\mathrm{SW}$ (Anticlockwise) & & & $\begin{array}{c}\mathrm{SAC} 2_{\mathrm{B}}+(180- \\
\left.\mathrm{SAC} 1_{\mathrm{B}}\right)\end{array}$ & \\
\hline $\mathrm{NE}($ Clockwise $)$ & \multirow{2}{*}{$\mathrm{NE}$} & \multirow{2}{*}{$\mathrm{NE}$} & $\begin{array}{l}\mathrm{SAC} 1_{\mathrm{A}}+(180- \\
\left.\mathrm{SAC} 2_{\mathrm{B}}\right)\end{array}$ & \multirow{2}{*}{ Abs $\left(S A C 1_{A}-S A C 1_{B}\right)$} \\
\hline $\mathrm{NE}($ Anticlockwise) & & & $\begin{array}{c}\mathrm{SAC} 2_{\mathrm{B}}+(180- \\
\left.\mathrm{SAC} 1_{\mathrm{B}}\right)\end{array}$ & \\
\hline $\mathrm{NE}$ & SE & $\mathrm{NE}$ & $\mathrm{SAC} 2_{\mathrm{B}}+\mathrm{SAC} 1_{\mathrm{A}}$ & $\operatorname{Abs}\left(\mathrm{SAC1}_{\mathrm{A}}-\mathrm{SAC1_{ \textrm {B } } )}\right.$ \\
\hline SE & $\mathrm{NE}$ & SE & $\mathrm{SAC} 2_{\mathrm{B}}+\mathrm{SAC} 1_{\mathrm{A}}$ & $\operatorname{Abs}\left(\mathrm{SAC1}_{\mathrm{A}}-\mathrm{SAC1} 1_{\mathrm{B}}\right)$ \\
\hline NW & SW & NW & $\mathrm{SAC} 2_{\mathrm{B}}+\mathrm{SAC} 1_{\mathrm{A}}$ & $\operatorname{Abs}\left(\mathrm{SAC1}_{\mathrm{A}}-\mathrm{SAC1} 1_{\mathrm{B}}\right)$ \\
\hline SW & NW & SW & $S A C 2_{B}+S A C 1_{A}$ & $\operatorname{Abs}\left(\mathrm{SAC1}_{\mathrm{A}}-\mathrm{SAC1} 1_{\mathrm{B}}\right)$ \\
\hline \multirow{2}{*}{ SE } & \multirow{2}{*}{ SW } & SE & \multirow{2}{*}{$\begin{array}{l}180-\left(\mathrm{SAC1}_{\mathrm{A}}+\right. \\
\left.\mathrm{SAC} 2_{\mathrm{B}}\right)\end{array}$} & $\begin{array}{c}\mathrm{Abs}\left(\mathrm{SAC} 1_{\mathrm{A}}{ }^{*}-\mathrm{SAC} 1_{\mathrm{A}}{ }^{* *}\right)- \\
\mathrm{SAC} 1_{\mathrm{B}}\end{array}$ \\
\hline & & SW & & $\begin{array}{c}\mathrm{SAC1}_{\mathrm{B}}+\mathrm{Abs}\left(\mathrm{SAC} 1_{\mathrm{A}}{ }^{*}-\right. \\
\left.\mathrm{SAC}_{\mathrm{A}}{ }^{* *}\right)\end{array}$ \\
\hline \multirow{2}{*}{ SW } & \multirow{2}{*}{ SE } & SW & \multirow{2}{*}{$\begin{array}{l}180-\left(\mathrm{SAC1}_{\mathrm{A}}+\right. \\
\left.\quad \mathrm{SAC} 2_{\mathrm{B}}\right)\end{array}$} & $\begin{array}{c}\mathrm{Abs}\left(\mathrm{SAC} 1_{\mathrm{A}}{ }^{*}-\mathrm{SAC} 1_{\mathrm{A}}{ }^{* *}\right)- \\
\mathrm{SAC} 1_{\mathrm{B}}\end{array}$ \\
\hline & & SE & & $\begin{array}{c}\mathrm{SAC1}_{\mathrm{B}}+\mathrm{Abs}\left(\mathrm{SAC} 1_{\mathrm{A}}{ }^{*}-\right. \\
\left.\mathrm{SAC}_{\mathrm{A}}{ }^{* *}\right)\end{array}$ \\
\hline \multirow{2}{*}{$\mathrm{NE}$} & \multirow{2}{*}{ NW } & $\mathrm{NE}$ & \multirow{2}{*}{$\begin{array}{l}180-\left(\mathrm{SAC1}_{\mathrm{A}}+\right. \\
\left.\mathrm{SAC} 2_{\mathrm{B}}\right)\end{array}$} & $\begin{array}{c}\mathrm{Abs}\left(\mathrm{SAC} 1_{\mathrm{A}}{ }^{*}-\mathrm{SAC} 1_{\mathrm{A}}{ }^{* *}\right)- \\
\mathrm{SAC} 1_{\mathrm{B}}\end{array}$ \\
\hline & & NW & & $\begin{array}{c}\mathrm{SAC}_{\mathrm{B}}+\mathrm{Abs}\left(\mathrm{SAC} 1_{\mathrm{A}}{ }^{*}-\right. \\
\left.\mathrm{SAC}_{\mathrm{A}}{ }^{* *}\right)\end{array}$ \\
\hline \multirow{2}{*}{ NW } & \multirow{2}{*}{$\mathrm{NE}$} & NW & \multirow{2}{*}{$\begin{array}{l}180-\left(\mathrm{SAC1}_{\mathrm{A}}+\right. \\
\left.\mathrm{SAC2} 2_{\mathrm{B}}\right)\end{array}$} & $\begin{array}{c}\mathrm{Abs}\left(\mathrm{SAC} 1_{\mathrm{A}}{ }^{*}-\mathrm{SAC} 1_{\mathrm{A}}{ }^{* *}\right)- \\
\mathrm{SAC} 1_{\mathrm{B}}\end{array}$ \\
\hline & & $\mathrm{NE}$ & & $\begin{array}{c}\mathrm{SAC1}_{\mathrm{B}}+\mathrm{Abs}\left(\mathrm{SAC} 1_{\mathrm{A}}{ }^{*}-\right. \\
\left.\operatorname{SAC}_{\mathrm{A}}{ }^{* *}\right)\end{array}$ \\
\hline
\end{tabular}

$S A C 1_{A}{ }^{*}$ and $S A C 1_{A}^{* *}$ represented as $S_{A Z}{ }^{*}$ and $S_{A Z}{ }^{* *}$ respectively in Theory \& Descriptions Section (Subsection-1).

Table 3: Determination of ' $v$ ' and ' $m$ ' Curved Azimuth departure course to Curved Azimuth departure course [SAME BEND DIRECTION]

\begin{tabular}{|c|c|c|c|c|}
\hline \multicolumn{5}{|c|}{ Azimuth Angles selection } \\
\hline $\begin{array}{c}\text { SAC1 } 1_{A}(\text { First } \\
\text { Trajectory Azimuth } \\
\text { Direction) - FROM }\end{array}$ & $\begin{array}{c}\mathrm{SAC} 2_{\mathrm{B}}(\text { Second } \\
\text { Trajectory Azimuth } \\
\text { Direction) - TO }\end{array}$ & $\begin{array}{l}\text { SAC1 }{ }_{\mathrm{B}} \text { (Azimuth } \\
\text { deviation of final } \\
\text { target from the } \\
\text { origin) }\end{array}$ & $\mathrm{V}$ & $\mathrm{m}$ \\
\hline $\mathrm{NE}(\mathrm{CW})$ & $\mathrm{NE}(\mathrm{CW})$ & $\mathrm{NE}$ & $\mathrm{SAC}_{\mathrm{A}}+\left(180-\mathrm{SAC} 2_{\mathrm{B}}\right)$ & Abs $\left(S A C 1_{A}-S A C 1_{B}\right)$ \\
\hline NE (ACW) & NE (ACW) & NE & $\mathrm{SAC} 2_{\mathrm{B}}+\left(180-\mathrm{SAC}_{\mathrm{B}}\right)$ & Abs $\left(S A C 1_{A}-S A C 1_{B}\right)$ \\
\hline NW (CW) & NW (CW) & NW & $\mathrm{SAC} 2_{\mathrm{B}}+\left(180-\mathrm{SAC}_{\mathrm{B}}\right)$ & Abs $\left(S A C 1_{A}-S A C 1_{B}\right)$ \\
\hline NW (ACW) & NW (ACW) & NW & $\mathrm{SAC}_{\mathrm{A}}+\left(180-\mathrm{SAC} 2_{\mathrm{B}}\right)$ & $\operatorname{Abs}\left(\mathrm{SAC1}_{\mathrm{A}}-\mathrm{SAC} 1_{\mathrm{B}}\right)$ \\
\hline $\mathrm{SW}(\mathrm{CW})$ & $\mathrm{SW}(\mathrm{CW})$ & SW & $S A C 1_{A}+\left(180-S A C 2_{B}\right)$ & $\operatorname{Abs}\left(\mathrm{SAC1}_{\mathrm{A}}-\mathrm{SAC} 1_{\mathrm{B}}\right)$ \\
\hline SW (ACW) & SW (ACW) & SW & $\mathrm{SAC} 2_{\mathrm{B}}+\left(180-\mathrm{SAC}_{\mathrm{B}}\right)$ & $\operatorname{Abs}\left(\mathrm{SAC1}_{\mathrm{A}}-\mathrm{SAC} 1_{\mathrm{B}}\right)$ \\
\hline $\mathrm{SE}(\mathrm{CW})$ & SE (CW) & SE & $\mathrm{SAC}_{\mathrm{B}}+\left(180-\mathrm{SAC}_{\mathrm{B}}\right)$ & $\operatorname{Abs}\left(\mathrm{SAC1}_{\mathrm{A}}-\mathrm{SAC} 1_{\mathrm{B}}\right)$ \\
\hline SE (ACW) & SE (ACW) & SE & $S A C 1_{A}+\left(180-S A C 2_{B}\right)$ & $\operatorname{Abs}\left(\mathrm{SAC1}_{\mathrm{A}}-\mathrm{SAC} 1_{\mathrm{B}}\right)$ \\
\hline $\mathrm{NE}(\mathrm{CW})$ & SE (CW) & $\mathrm{NE}$ & $\mathrm{SAC} 1_{\mathrm{A}}+\mathrm{SAC} 2_{\mathrm{B}}$ & $\operatorname{Abs}\left(\mathrm{SAC1}_{\mathrm{A}}-\mathrm{SAC} 1_{\mathrm{B}}\right)$ \\
\hline $\mathrm{NE}(\mathrm{CW})$ & SE (CW) & SE & $\mathrm{SAC} 1_{\mathrm{A}}+\mathrm{SAC} 2_{\mathrm{B}}$ & $180-\left(\mathrm{SAC1}_{\mathrm{A}}+\mathrm{SAC} 1_{\mathrm{B}}\right)$ \\
\hline NE (ACW) & NW (ACW) & NW & $180-\left(\mathrm{SAC1}_{\mathrm{A}}+\mathrm{SAC} 2_{\mathrm{B}}\right)$ & $\mathrm{SAC1}_{\mathrm{A}}+\mathrm{SAC} 1_{\mathrm{B}}$ \\
\hline NE (ACW) & NW (ACW) & $\mathrm{NE}$ & $\mathrm{SAC} 2_{\mathrm{B}}+\left(180-\mathrm{SAC}_{\mathrm{B}}\right)$ & $\operatorname{Abs}\left(\mathrm{SAC1}_{\mathrm{A}}-\mathrm{SAC} 1_{\mathrm{B}}\right)$ \\
\hline NW (ACW) & SW (ACW) & SW & $\mathrm{SAC} 1_{\mathrm{A}}+\mathrm{SAC} 2_{\mathrm{B}}$ & $180-\left(\mathrm{SAC1}_{\mathrm{A}}+\mathrm{SAC1} 1_{\mathrm{B}}\right)$ \\
\hline NW (ACW) & SW (ACW) & NW & $S A C 1_{A}+S A C 2_{B}$ & $\operatorname{Abs}\left(\mathrm{SAC1}_{\mathrm{A}}-\mathrm{SAC} 1_{\mathrm{B}}\right)$ \\
\hline $\mathrm{NW}(\mathrm{CW})$ & $\mathrm{NE}(\mathrm{CW})$ & $\mathrm{NE}$ & $180-\left(\mathrm{SAC1}_{\mathrm{A}}+\mathrm{SAC2} 2_{\mathrm{B}}\right)$ & $\mathrm{SAC} 1_{\mathrm{A}}+\mathrm{SAC} 1_{\mathrm{B}}$ \\
\hline $\mathrm{NW}(\mathrm{CW})$ & $\mathrm{NE}(\mathrm{CW})$ & NW & $180-\left(\mathrm{SAC1}_{\mathrm{A}}+\mathrm{SAC2} 2_{\mathrm{B}}\right)$ & $\operatorname{Abs}\left(S A C 1_{A}-S A C 1_{B}\right)$ \\
\hline
\end{tabular}


Directional Well Trajectory Design: The Theoretical Development of Azimuth BendS AND TuRnS.... P. o. okpozo, et al

\begin{tabular}{|c|c|c|c|c|}
\hline \multicolumn{5}{|c|}{ Azimuth Angles selection } \\
\hline $\begin{array}{c}\text { SAC } 1_{A}(\text { First } \\
\text { Trajectory Azimuth } \\
\text { Direction) - FROM }\end{array}$ & $\begin{array}{c}\mathrm{SAC} 2_{\mathrm{B}}(\text { Second } \\
\text { Trajectory Azimuth } \\
\text { Direction })-\mathrm{TO}\end{array}$ & $\begin{array}{l}\mathrm{SAC}_{\mathrm{B}}(\text { Azimuth } \\
\text { deviation of final } \\
\text { target from the } \\
\text { origin) }\end{array}$ & V & $\mathrm{m}$ \\
\hline SW (CW) & NW (CW) & SW & $\mathrm{SAC} 1_{\mathrm{A}}+\left(180-\mathrm{SAC} 2_{\mathrm{B}}\right)$ & $\operatorname{Abs}\left(S A C 1_{A}-S A C 1_{B}\right)$ \\
\hline $\mathrm{SW}(\mathrm{CW})$ & NW (CW) & NW & $\mathrm{SAC} 1_{\mathrm{A}}+\mathrm{SAC} 2_{\mathrm{B}}$ & $180-\left(S A C 1_{A}+S A C 1_{B}\right)$ \\
\hline SW (ACW) & SE (ACW) & SE & $180-\left(S A C 1_{A}+S A C 2_{B}\right)$ & $S A C 1_{A}+S A C 1_{B}$ \\
\hline SW (ACW) & SE (ACW) & SW & $180-\left(\mathrm{SAC} 1_{\mathrm{A}}+\mathrm{SAC} 2_{\mathrm{B}}\right)$ & $\operatorname{Abs}\left(S A C 1_{A}-S A C 1_{B}\right)$ \\
\hline $\mathrm{SE}(\mathrm{CW})$ & SW (CW) & SW & $180-\left(\mathrm{SAC} 1_{\mathrm{A}}+\mathrm{SAC} 2_{\mathrm{B}}\right)$ & $S A C 1_{A}+S A C 1_{B}$ \\
\hline SE (CW) & SW (CW) & SE & $180-\left(\mathrm{SAC} 1_{\mathrm{A}}+\mathrm{SAC} 2_{\mathrm{B}}\right)$ & $\operatorname{Abs}\left(S A C 1_{A}-S A C 1_{B}\right)$ \\
\hline SE (ACW) & NE (ACW) & SE & $\mathrm{SAC} 1_{\mathrm{A}}+\mathrm{SAC} 2_{\mathrm{B}}$ & $\operatorname{Abs}\left(S A C 1_{A}-S A C 1_{B}\right)$ \\
\hline SE (ACW) & NE (ACW) & NE & $S A C 1_{A}+S A C 2_{B}$ & $180-\left(\mathrm{SAC} 1_{\mathrm{A}}+\mathrm{SAC} 1_{\mathrm{B}}\right)$ \\
\hline
\end{tabular}

Table 4: Determination of ' $V$ ' and ' $m$ ' Curved Azimuth departure course to Curved Azimuth departure course [OPPOSITE BEND DIRECTION]

\begin{tabular}{|c|c|c|c|c|}
\hline \multicolumn{5}{|c|}{ Azimuth Angles selection } \\
\hline $\begin{array}{c}\text { SAC1 } 1_{A}(\text { First } \\
\text { Trajectory Azimuth } \\
\text { Direction) - FROM }\end{array}$ & $\begin{array}{c}\text { SAC2 }_{\mathrm{B}}(\text { Second } \\
\text { Trajectory Azimuth } \\
\text { Direction })- \text { TO }\end{array}$ & $\begin{array}{c}\mathrm{SAC} 1_{\mathrm{B}}(\text { Azimuth } \\
\text { deviation of final } \\
\text { target from the origin })\end{array}$ & V & $\mathrm{m}$ \\
\hline \multirow{2}{*}{$\mathrm{NE}(\mathrm{CW})$} & \multirow{2}{*}{$\mathrm{NE}(\mathrm{ACW})$} & $\mathrm{NE}\left(\mathrm{SAC} 1_{\mathrm{A}}>\mathrm{SAC} 1_{\mathrm{B}}\right)$ & $\mathrm{SAC2}_{\mathrm{B}}+\left(180-\mathrm{SAC1}_{\mathrm{B}}\right)$ & \multirow{16}{*}{$\operatorname{Abs}\left(S A C 1_{A}-S A C 1_{B}\right)$} \\
\hline & & $\operatorname{NE}\left(S A C 1_{A}<S A C 1_{B}\right)$ & $\mathrm{SAC1}_{\mathrm{A}}+\left(180-\mathrm{SAC} 2_{\mathrm{B}}\right)$ & \\
\hline \multirow{2}{*}{ SW (ACW) } & \multirow{2}{*}{$\mathrm{SW}(\mathrm{CW})$} & $\mathrm{SW}\left(\mathrm{SAC} 1_{\mathrm{A}}<\mathrm{SAC} 1_{\mathrm{B}}\right)$ & $\mathrm{SAC1}_{\mathrm{A}}+\left(180-\mathrm{SAC} 2_{\mathrm{B}}\right)$ & \\
\hline & & $\mathrm{SW}\left(\mathrm{SAC1}_{\mathrm{A}}>\mathrm{SAC} 1_{\mathrm{B}}\right)$ & $\mathrm{SAC}_{\mathrm{B}}+\left(180-\mathrm{SAC} 1_{\mathrm{B}}\right)$ & \\
\hline \multirow{2}{*}{ SE (CW) } & \multirow{2}{*}{ SE (ACW) } & $\mathrm{SE}\left(\mathrm{SAC} 1_{\mathrm{A}}<\mathrm{SAC} 1_{\mathrm{B}}\right)$ & $\mathrm{SAC1}_{\mathrm{A}}+\left(180-\mathrm{SAC} 2_{\mathrm{B}}\right)$ & \\
\hline & & $\mathrm{SE}\left(\mathrm{SAC1}_{\mathrm{A}}>\mathrm{SAC1} 1_{\mathrm{B}}\right)$ & $\mathrm{SAC2}_{\mathrm{B}}+\left(180-\mathrm{SAC} 1_{\mathrm{B}}\right)$ & \\
\hline \multirow{2}{*}{ SE (ACW) } & \multirow{2}{*}{$\mathrm{SE}(\mathrm{CW})$} & $\mathrm{SE}\left(\mathrm{SAC1} 1_{\mathrm{A}}<\mathrm{SAC} 1_{\mathrm{B}}\right)$ & $\mathrm{SAC} 2_{\mathrm{B}}+\left(180-\mathrm{SAC} 1_{\mathrm{B}}\right)$ & \\
\hline & & $\mathrm{SE}\left(\mathrm{SAC1} 1_{\mathrm{A}}>\mathrm{SAC1} 1_{\mathrm{B}}\right)$ & $\mathrm{SAC}_{\mathrm{A}}+\left(180-\mathrm{SAC} 2_{\mathrm{B}}\right)$ & \\
\hline \multirow{2}{*}{ NW (ACW) } & \multirow{2}{*}{$\mathrm{NW}(\mathrm{CW})$} & $\mathrm{NW}\left(\mathrm{SAC} 1_{\mathrm{A}}<\mathrm{SAC} 1_{\mathrm{B}}\right)$ & $\mathrm{SAC1}_{\mathrm{A}}+\left(180-\mathrm{SAC} 2_{\mathrm{B}}\right)$ & \\
\hline & & $\mathrm{NW}\left(\mathrm{SAC} 1_{\mathrm{A}}>\mathrm{SAC} 1_{\mathrm{B}}\right)$ & $\mathrm{SAC} 2_{\mathrm{B}}+\left(180-\mathrm{SAC}_{\mathrm{B}}\right)$ & \\
\hline \multirow{2}{*}{ SW (CW) } & \multirow{2}{*}{ SW (ACW) } & $\mathrm{SW}\left(\mathrm{SAC} 1_{\mathrm{A}}<\mathrm{SAC} 1_{\mathrm{B}}\right)$ & $\mathrm{SAC}_{\mathrm{B}}+\left(180-\mathrm{SAC} 1_{\mathrm{B}}\right)$ & \\
\hline & & $\mathrm{SW}\left(\mathrm{SAC1}_{\mathrm{A}}>\mathrm{SAC} 1_{\mathrm{B}}\right)$ & $\mathrm{SAC1}_{\mathrm{A}}+\left(180-\mathrm{SAC} 2_{\mathrm{B}}\right)$ & \\
\hline \multirow{2}{*}{$\mathrm{NW}(\mathrm{CW})$} & \multirow{2}{*}{ NW (ACW) } & $\mathrm{NW}\left(\mathrm{SAC} 1_{\mathrm{A}}<\mathrm{SAC} 1_{\mathrm{B}}\right)$ & $\mathrm{SAC}_{\mathrm{A}}+\left(180-\mathrm{SAC} 2_{\mathrm{B}}\right)$ & \\
\hline & & $\mathrm{NW}\left(\mathrm{SAC} 1_{\mathrm{A}}>\mathrm{SAC} 1_{\mathrm{B}}\right)$ & $\mathrm{SAC}_{\mathrm{B}}+\left(180-\mathrm{SAC} 1_{\mathrm{B}}\right)$ & \\
\hline \multirow{2}{*}{$\mathrm{N}(\mathrm{ACW})$} & \multirow{2}{*}{$\mathrm{NE}(\mathrm{CW})$} & $\mathrm{NE}\left(\mathrm{SAC} 1_{\mathrm{A}}>\mathrm{SAC} 1_{\mathrm{B}}\right)$ & $\mathrm{SAC}_{\mathrm{B}}+\left(180-\mathrm{SAC} 1_{\mathrm{B}}\right)$ & \\
\hline & & $\mathrm{NE}\left(\mathrm{SAC} 1_{\mathrm{A}}<\mathrm{SAC} 1_{\mathrm{B}}\right)$ & $\mathrm{SAC}_{\mathrm{A}}+\left(180-\mathrm{SAC} 2_{\mathrm{B}}\right)$ & \\
\hline
\end{tabular}

Table 5: Complex trajectory data input (WellTIT v. 1.0)

\begin{tabular}{lcccccc}
\hline $\begin{array}{l}\text { Trajectory } \\
\text { type }\end{array}$ & $\begin{array}{c}\text { Total vertical } \\
\text { Depth (ft) }\end{array}$ & $\begin{array}{c}\text { Kick-Off } \\
\text { point (ft) }\end{array}$ & $\begin{array}{c}\text { Horizontal } \\
\text { departure }(\mathrm{ft})\end{array}$ & $\begin{array}{c}\text { Inclination angle } \\
\text { (degree) }\end{array}$ & $\begin{array}{c}\text { Azimuth } \\
\text { Direction }\end{array}$ & $\begin{array}{c}\text { Bend } \\
\text { Direction }\end{array}$ \\
\hline $\begin{array}{l}\text { Build \& Hold } \\
\begin{array}{l}\text { Straight } \\
\text { course }\end{array}\end{array}$ & 7000 & 3000 & 1100 & 25 & $30^{\circ}$ NE & Clockwise \\
\hline
\end{tabular}

\section{APPLICATION OF THEORY AND PROCESSES}

\subsection{Theoretical Example by Application Using Welltit V.} 1.0

WellTIT version 1.0 is a self-developed application meant for designing various well trajectories. Though is at its early development stage, it has the ability to resolve some technical data input by utilizing various complex mathematics inbuilt in the program. The application was developed using the Microsoft visual studio, hence it possesses its own graphic user interface and the survey records and graphical plots are transferred to a Microsoft excel workbook where it can be saved for record keeping.

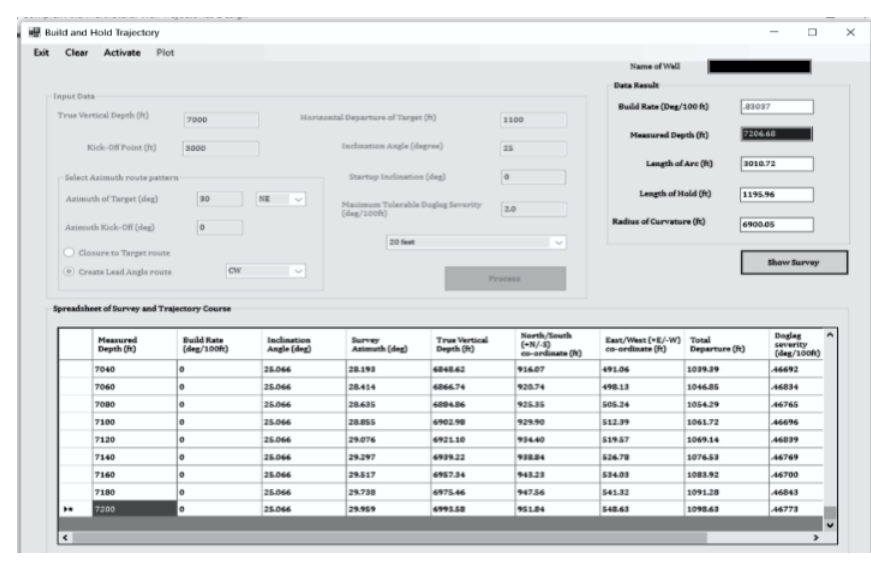

Figure 4: Build-and-Hold trajectory calculation page 
The data input is shown below (Table 5) and Figures (4 to 8) captures the application, results and plots of a complex well trajectory using option (E) theoretical design of figure (3) as an example. In this example we assumed zero delineation (no magnetic reference), no possible magnetic interferences and ideal BHA configuration. The complex well trajectory to be designed comprises of a Build and Hold trajectory and a Straight course. The set maximum tolerable dogleg severity selected was $3.0^{\circ} / 100 \mathrm{ft}$. The survey record is shown in Table 6.

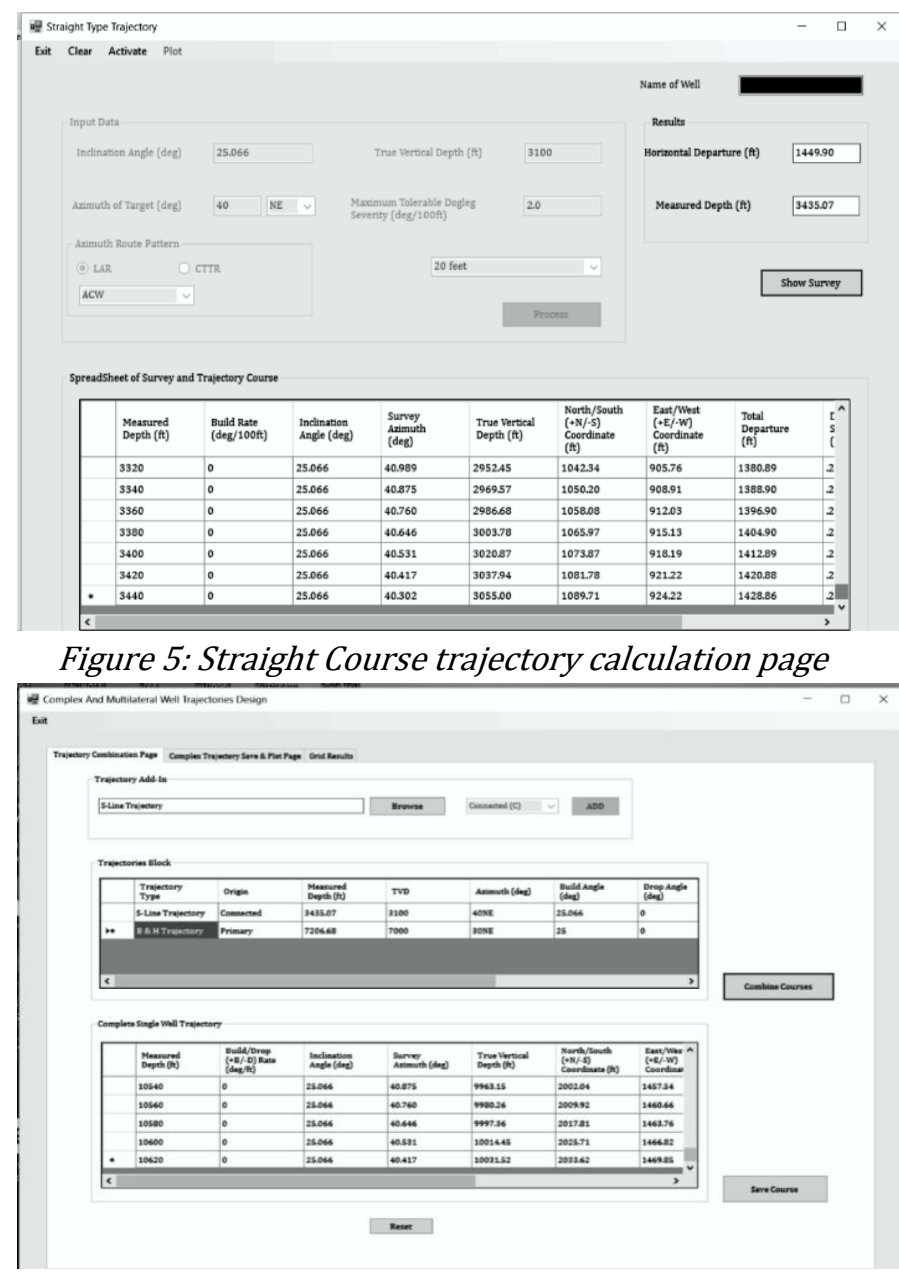

Figure 6: Trajectories Combination page

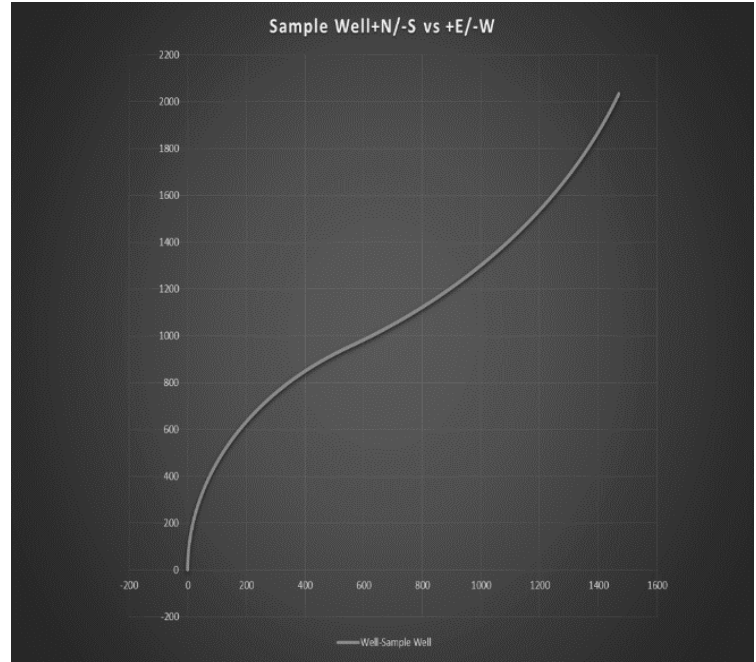

Figure 7: Northing vs Easting plot of sample well

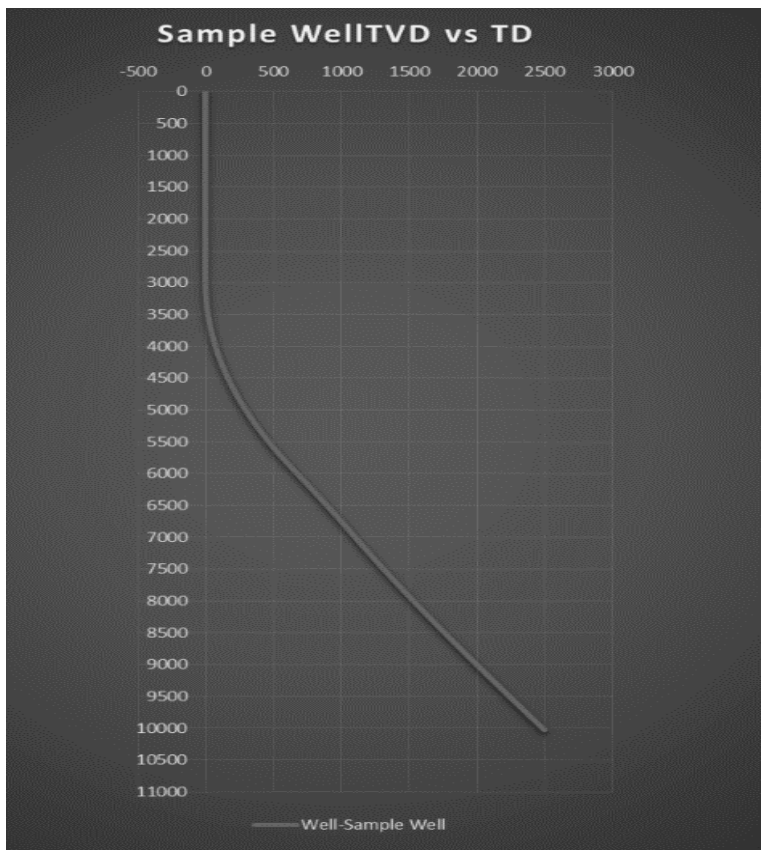

Figure 8: TVD vs Hor. Dep. of sample well

Table 6: Summary of Survey record for complex well trajectory example using WellTIT v. 1.0

\begin{tabular}{cccccc}
\hline $\begin{array}{c}\text { WELL } \\
\text { NAME }\end{array}$ & $\begin{array}{c}\text { Well } \\
\text { Xristics } \\
\text { (Basic/Co } \\
\text { mplex) }\end{array}$ & $\begin{array}{c}\text { No. of } \\
\text { Basic } \\
\text { Traj.(s) }\end{array}$ & $\begin{array}{c}\text { Measured } \\
\text { Depth }\end{array}$ & $\begin{array}{c}\text { True } \\
\text { Vertical } \\
\text { Depth }\end{array}$ & $\begin{array}{c}\text { Survey } \\
\text { Azimuth of } \\
\text { Target }\end{array}$ \\
\hline $\begin{array}{c}\text { Sample } \\
\text { Well }\end{array}$ & Complex & 2 & $10620 \mathrm{ft}$ & $10031.52 \mathrm{ft}$ & 40NEdeg \\
\hline \hline
\end{tabular}




\begin{tabular}{|c|c|c|c|c|c|c|c|c|c|}
\hline $\begin{array}{l}\text { Measured } \\
\text { Depth (ft) }\end{array}$ & $\begin{array}{c}\text { +Build/- } \\
\text { Drop Rate } \\
\text { (deg/100ft) }\end{array}$ & $\begin{array}{l}\text { Inclination } \\
\text { Angle } \\
\text { (deg) }\end{array}$ & $\begin{array}{l}\text { Survey } \\
\text { Azimuth } \\
\text { (deg) }\end{array}$ & $\begin{array}{c}\text { True } \\
\text { Vertical } \\
\text { Depth (ft) }\end{array}$ & $\begin{array}{c}\text { North/Sout } \\
\mathrm{h}(+\mathrm{N} /-\mathrm{S}) \\
\text { Cordinate } \\
(\mathrm{ft})\end{array}$ & $\begin{array}{c}\text { East/West( } \\
+\mathrm{E} / \mathrm{-W}) \\
\text { Cordinate } \\
(\mathrm{ft})\end{array}$ & $\begin{array}{c}\text { Total } \\
\text { Departure } \\
\text { (ft) }\end{array}$ & $\begin{array}{c}\text { Dogleg } \\
\text { Severity } \\
\text { (deg/100ft) }\end{array}$ & $\begin{array}{c}\text { Tool Face } \\
\text { Angle } \\
\text { (deg) }\end{array}$ \\
\hline 0 & 0 & 0 & 0 & 0 & 0 & 0 & 0 & 0 & $\mathrm{NaN}$ \\
\hline 20 & 0 & 0 & 0 & 20 & 0 & 0 & 0 & 0 & $\mathrm{NaN}$ \\
\hline 40 & 0 & 0 & 0 & 40 & 0 & 0 & 0 & 0 & $\mathrm{NaN}$ \\
\hline 3000 & 0 & 0 & 0 & 3000 & 0 & 0 & 0 & 0 & $\mathrm{NaN}$ \\
\hline 3020 & 0.83037 & 0.166 & 0.001 & 3020 & 0.03 & 0 & 0.03 & 0.8304 & $\mathrm{NaN}$ \\
\hline 3040 & 0.83037 & 0.332 & 0.003 & 3040 & 0.12 & 0 & 0.12 & 0.8304 & 0.004 \\
\hline 6000 & 0.83037 & 24.9 & 16.719 & 5906.381 & 606.14 & 182.07 & 632.89 & 0.9491 & 29.0 \\
\hline 6020 & 0.83037 & 25.066 & 16.939 & 5924.508 & 613.17 & 186.75 & 640.98 & 0.9516 & 29.34 \\
\hline 6040 & 0 & 25.066 & 17.16 & 5942.62 & 620.19 & 191.5 & 649.08 & 0.4676 & $\mathrm{NaN}$ \\
\hline 6060 & 0 & 25.066 & 17.38 & 5960.74 & 627.17 & 196.31 & 657.17 & 0.467 & $\mathrm{NaN}$ \\
\hline 10580 & 0 & 25.066 & 40.646 & 9997.36 & 2017.81 & 1463.76 & 2479.162 & 0.2416 & 98.78 \\
\hline 10600 & 0 & 25.066 & 40.531 & 10014.45 & 2025.71 & 1466.82 & 2487.536 & 0.2416 & 98.83 \\
\hline 10620 & 0 & 25.066 & 40.417 & 10031.52 & 2033.62 & 1469.85 & 2495.907 & 0.2416 & 98.88 \\
\hline
\end{tabular}

\section{CONCLUSION}

The theory created proved to work effectively without any hitch in the final survey output and trajectory graphical plot. The theoretical equations being actually imbedded in the WellTIT program can of course being used to run further imagined complex trajectory courses. The entire record on dogleg severity was less than the set maximum tolerable value. The challenges with using WellTIT are;

1) That the trajectory has to be properly imagined and practically drawn, hence coming up with vital data input.

2) Data on the geomagnetic reference from the BGS is expensive to obtain for trajectory calculation accuracy improvement.

Nevertheless, this resolved theory will serve as resource material to educate students in the area of Complex directional well trajectory design.

\section{REFERENCES}

[1] Bourgoyne Adam T. Jr., Millheim Keith K., Chenevert Martin E., Young F. S. Jr. Applied Drilling Engineering. Society of Petroleum Engineers, Richardson,. Texas, pp. 351-66. 1986.
[2] Rogers, W. M. Hole Deviation and Horizontal Drilling. IADC drilling manual, , eleventh version. 2000.

[3] Stromhaug Andreas Holm. "Directional Drilling Advance Trajectory Modelling". Published Masters' Thesis, NTNU - Trondhum Norwegian University of Science and Technology, 2014.

[4] Eustes III Alfred W. Directional Drilling Seminar. Colorado school of mines Golden, , Colorado. 2001.

[5] Evers Jack F. Directional drilling and deviation control. Applied Drilling Engineering, , $19^{\text {th }}$ printing. (1984).

[6] Craig, J. T. Jr. and Randall, B. V. Directional Drilling Survey Calculation. Pet. Eng., 1976.

[7] Farah Omar Farah 'Directional well design, Trajectory and survey calculations, with a case study in Fiale, Asal rift, Djibouti'. Geothermal Training Programme - number 27, United Nations University, Iceland, 2013.

[8] British Geological Survey (BGS) Global Geomagnetic Model, www.geomag.bgs.ac.uk, 2014.

[9] Amorin R., Broni-Bediako E. "Application of minimum Curvature method to wellpath Calculations". Research Journal of Applied Sciences Engineering and Technology, 2 (7), pp. 679-686, 2010.

\section{NOMENCLATURE:}

$\Theta_{1-2} \quad$ Inclination angle change from point 1 to 2 (degrees)

$\emptyset_{1-2} \quad$ Azimuth angle change from point 1 to 2 (degrees)

$\mathrm{HD}_{\text {STR }} \quad$ Straight line course Horizontal departure (feet)

$\mathrm{HD}_{\text {CURV }} \quad$ Curved line course Horizontal departure (feet)

$\mathrm{R}_{\mathrm{AZ}} \quad$ Azimuth radius of curvature (feet)

$\mathrm{S}_{\mathrm{AZ}} \quad$ Azimuth angle deviation from reference point (degrees)

$\mathrm{X}_{\mathrm{NW}} \quad$ Overall azimuth angle change in North-West direction

$\mathrm{S}_{\mathrm{AZ}}{ }^{*} \quad$ Azimuth angle startup deviation from any chosen coordinate reference point (degrees)

$\mathrm{S}_{\mathrm{AZ}}^{* *} \quad$ Final azimuth deviation angle from any chosen coordinate reference point (degrees) 
$\mathrm{T}_{\mathrm{gt}}$

0

SAC1 $1_{A}$

$S A C 1_{B}$

SAC2 ${ }_{B}$

SAC2 ${ }_{A}$

$\mathrm{T}_{\mathrm{A}}$

$\mathrm{T}_{\mathrm{B}}$

$\mathrm{T}_{\mathrm{T}}$

E

$\mathrm{N}$

S

W

NW

NE

SW

SE

$\mathrm{V}$

M

Abs

ACW

CW
Well course Target

Origin point (kick off point)

Azimuth angle direction of first trajectory course from point 0.

Azimuth angle direction of second trajectory course from point 0 .

Azimuth angle direction of second trajectory course from point, $\mathrm{T}_{\mathrm{A}}$

Azimuth angle direction created by tangent of first trajectory course

Target point of first trajectory course

Target point of second trajectory course

Tangent of first trajectory course

East coordinate direction

North coordinate direction

South coordinate direction

West coordinate direction

North-West coordinate direction

North-East coordinate direction

South-West coordinate direction

South-East coordinate direction

The angle created in Triangle $\Delta O \mathrm{~T}_{\mathrm{A}} \mathrm{T}_{\mathrm{B}}$ by difference between $\mathrm{SAC} 1_{\mathrm{A}}$ and $\mathrm{SAC} \mathrm{B}_{\mathrm{B}}$

The resolved angle at $0{\widehat{T_{A}}}_{\mathrm{T}_{\mathrm{B}}}$

Absolute value

Anticlockwise direction

Clockwise direction 